\title{
Werkzeuge zur sauberen Fasertrennung in CFK-Bauteilen
}

Wegen ihrer guten Leichtbaueigenschaften sind mit Kohlefasern verstärkte Kunststoffe (CFK) stark verbreitet. Moderne Flugzeuge, wie etwa der Airbus A350, können zu über $50 \%$ aus neuen Materialien bestehen, wobei CFK den Löwenanteil ausmacht. Nach Angaben des Kompetenznetzwerkes Carbon Composites e.V. (CCeV) wurden 2011 weltweit bereits rund 55.000 t CFK verarbeitet. Bis 2015 erwartet der CCeV nahezu eine Verdoppelung auf etwa 100.000 t. Wie global dieser Trend ist, zeigt eine weitere Zahl, die Prof. Uwe Heisel, Direktor des Instituts für Werkzeugmaschinen (IfW) der Universität Stuttgart, bei seinem Eingangsreferat auf der 2. IfW-Tagung vorstellte: Laut CCeV verteilte sich der Bedarf an CFK 2011 nahezu gleichmäßig auf Westeuropa (34\%), Nordamerika (36\%) und Asien (etwa $30 \%$ ). Wesentliche Unterschiede im technologischen Standard sieht Heisel zwischen den Regionen nicht.

Gleichzeitig gilt die Bearbeitung von mit Kohlefasern verstärkten Kunststoffen (CFK) laut Heisel weiterhin „als nicht unproblematisch und ist daher ein stark diskutiertes Thema". Trotz aller Fortschritte bleibe es eine Herausforderung, Werkzeuge zur sauberen Fasertrennung zu entwickeln. Er wies zusätzlich auf unzureichend geklärte Gefahren bei der spanenden CFK-Bearbeitung hin, insbesondere auf lungengängige Stäube und deren Absaugung. Im Hinblick auf bekannte Phänomene, wie Delamination beim Bohreraustritt sowie Gratbildung und Ausfransung beim Bohren und Fräsen, betonte der IfWDirektor den bestehenden Handlungsbedarf. Es gelte, die Schnittparameter für eine wirksame Zerspanung ohne Zerstörung der Matrix weiter zu erforschen. Nicht zuletzt fehlten bislang auch klare Definitionen zulässiger Schäden.

Benötigt werden neue Materialmodelle für die schnellere Simulation der Zerspanung, vor allem aber Fortschritte bei Werkzeugen und Maschinen. Als Beispiel nannte Heisel hybride CFK-Fräsmaschinen mit Ultraschallunterstützung, die geringere Bearbeitungs-

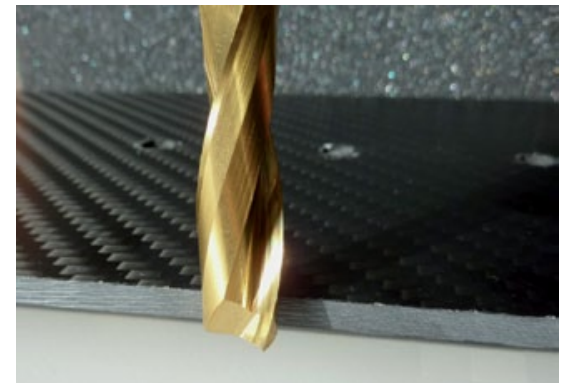

kräfte erfordern. Wegen des insgesamt großen Forschungsbedarfs wird am FraunhoferInstitut für Produktionstechnik und Automatisierung (IPA) in Stuttgart derzeit im Arbeitsgebiet "Prozesstechnologie" eine neue Abteilung für Leichtbautechnologien unter der Leitung von Dr. Marco Schneider aufgebaut. Bis 2016 sollen hier an die 50 Mitarbeiter beschäftigt sein. Der Schwerpunkt wird auf den Bearbeitungstechniken für Verbundmaterialien liegen.

Praxisberichte auf der Tagung zeigten konkrete Fortschritte, etwa bei der Werkzeugentwicklung auf. Die Spanne der vorgestellten Lösungen und Entwicklungen reichte vom halbautomatischen Bohren mit schnellem Umschalten von Vorschub und Drehzahl je nach erreichter Materialschicht in Mehrstoffverbunden über das Vibrationsbohren in CFK-Titanverbünden bis zu neuen Zerspanungssystemen. Unter anderem berichtete Ralph Hufschmied, Gründer des gleichnamigen Unternehmens, über Verschleißmechanismen bei Faserverbundwerkstoffen. Eine neue Beschichtung mit nanokristallinem Diamant (Korngröße 0,02 um) für CFK-Hartmetallfräser ermöglicht nach seinen Ausführungen schärfere Schneidkanten, längere Standzeiten und bessere Oberflächengüten. Hufschmied betonte, die Auswahl geeigneter Werkstoffe und Geometrien bei Fräsern sei deshalb so komplex, weil das Verhalten der Werkzeuge stark von der jeweils gewählten Vorschubkraft und-geschwindigkeit abhänge. Auch Dipl.-Wirtsch.-Ing. Tobias Pfeifroth vom IfW betonte die grundsätzliche Bedeu- tung von Beschichtungen. Neben der insgesamt wichtigen Werkzeuggeometrie hätten gerade Beschichtungen Einfluss auf Vorschubkraft und Delamination, so Pfeifroth.

Keramische Composites versprechen ebenfalls Fortschritte in der CFK-Zerspanung. Dr. Frank Kern vom Institut für Fertigungstechnologie keramischer Bauteile (IFKB) der Universität Stuttgart referierte über neue Keramikformulierungen, mit denen im Spritzguss endkonturnahe Fräsergeometrien erzeugt werden. Nach dem Sintern entstehen so hochfeste und hochzähe Werkstoffe. Unter der Leitung von Prof. Rainer Gadow wurden am IFKB außerdem Hochleistungskeramiken auf Basis von Aluminiumoxid mit Verstärkungsphase entwickelt und erprobt, die durch Funkenerosion endbearbeitet werden können. Eine Besonderheit dabei: Beim Erodieren entsteht nach Aussage von Frank Kern keine Aufsprödungszone.

Über ähnliche Fortschritte bei keramischen Werkzeugen zur CFK-Zerspanung berichtete Dr. Wolfgang Burger von Oximatec. Mit der von ihm vorgestellten Aluminiumoxidmatrix mit homogen verteiltem tetragonalem Zirkoniumdioxid und hexagonalen Plättchen (ZPTA) ließen sich Werkzeugbruchzähigkeiten von bis zu 7,8 MPa erreichen. In Praxisberichten, wie etwa von der EADS-Tochter Premium Aerotec in Augsburg und von Airbus Operations in Stade dagegen wurden durchweg Hartmetallwerkzeuge erwähnt. Hier ist also für die kommenden Jahre mit Bewegung und neuen Ergebnissen zu rechnen.

Ein laut Aussage von Dr. Marco Schneider vom Fraunhofer-Institut IPA bislang vernachlässigter Aspekt der CFK-Zerspanung sei die Absaugung an der Maschine. Nach seiner Aussage ist sie aktuell für etwa $45 \%$ des elektrischen Energieverbrauchs einer vergleichbaren Holzbearbeitungsmaschine verantwortlich. Vor allem durch werkzeugnahe Absaugung ließen sich laut Schneider bis zu $70 \%$ dieser Energie einsparen und gleichzeitig sauberere Werkstücke erzielen. 